\title{
Single-particle plasmon voltammetry (spPV) for detecting anion adsorption
}

Chad P. Byers, ${ }^{1,2}$ Benjamin S. Hoener, ${ }^{2}$ Wei-Shun Chang, ${ }^{2}$ Stephan Link, ${ }^{1,2,3}$ Christy F. Landes $^{1,2,3, *}$

${ }^{1}$ Smalley-Curl Institute Applied Physics Program, Rice University, Houston, Texas 77005, United States

${ }^{2}$ Dept. of Chemistry, Rice University, Houston, Texas 77005, United States

${ }^{3}$ Dept. of Electrical and Computer Engineering, Rice University, Houston, Texas 77005, United States

* Corresponding Author: cflandes@ rice.edu

Supporting Information includes:

Figures $\mathrm{S} 1$ to $\mathrm{S} 9$ 


\section{Table of Contents}

\section{Additional figures}

Figure S1: Cyclic voltammogram of $100 \mathrm{mM} \mathrm{Na}_{2} \mathrm{SO}_{4}$ in cells built with

ITO and Au thin film substrates.

Figure S2: Normalized transmission spectrum of a glass slide compared to electrochemical cells built using ITO or Au thin film working electrodes.

Figure S3: Transmission spectrum of electrochemical cell built with Au film and $100 \mathrm{mM} \mathrm{NaSO}_{4}$ as a function of applied potential.

Figure S4: Scattering spectrum of dimer on Au film during CV in $100 \mathrm{mM} \mathrm{Na}_{2} \mathrm{SO}_{4}$.

Figure S5: Scattering spectrum of Au film background during CV in $100 \mathrm{mM} \mathrm{Na} \mathrm{SO}_{4}$.

Figure S6: Comparison between spPV signals of dimer on $\mathrm{Au}$ film in

$100 \mathrm{mM} \mathrm{NaC} \mathrm{H}_{3} \mathrm{O}_{2}$, pH 8.5 vs $\mathrm{pH} 12$.

Figure S7: Sample spPV trace from $\mathrm{F}^{-}$control experiment compared to spPV response to sulfate adsorption.

Figure S8: Comparison between spPV signals derived from SB mode intensity vs integrated scattering intensity.

Figure S9: Correlated SEM with scattering spectra for monomers and dimers. 

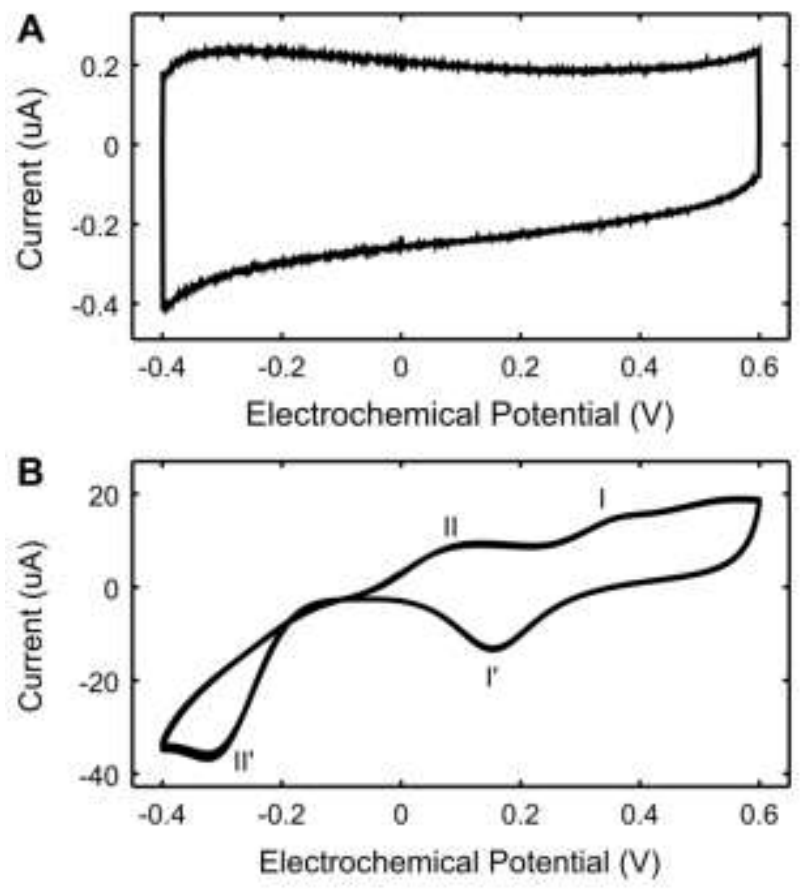

Figure S1. Cyclic voltammogram of $100 \mathrm{mM} \mathrm{Na}_{2} \mathrm{SO}_{4}$ in cells built with (A) ITO substrate, (B) Au film substrate with nanosphere monomers and dimers. Note that the current generated on the Au film working electrode is two orders of magnitude larger than that of the ITO working electrode. Because the density of nanoparticles is nearly identical, it is safe to assume that most of the current signal is generated from the substrate. In the gold film sample CV (B), the waves for sulfate adsorption and desorption are labeled I and I' along with peaks corresponding to hydrogen evolution reaction II and II'. 

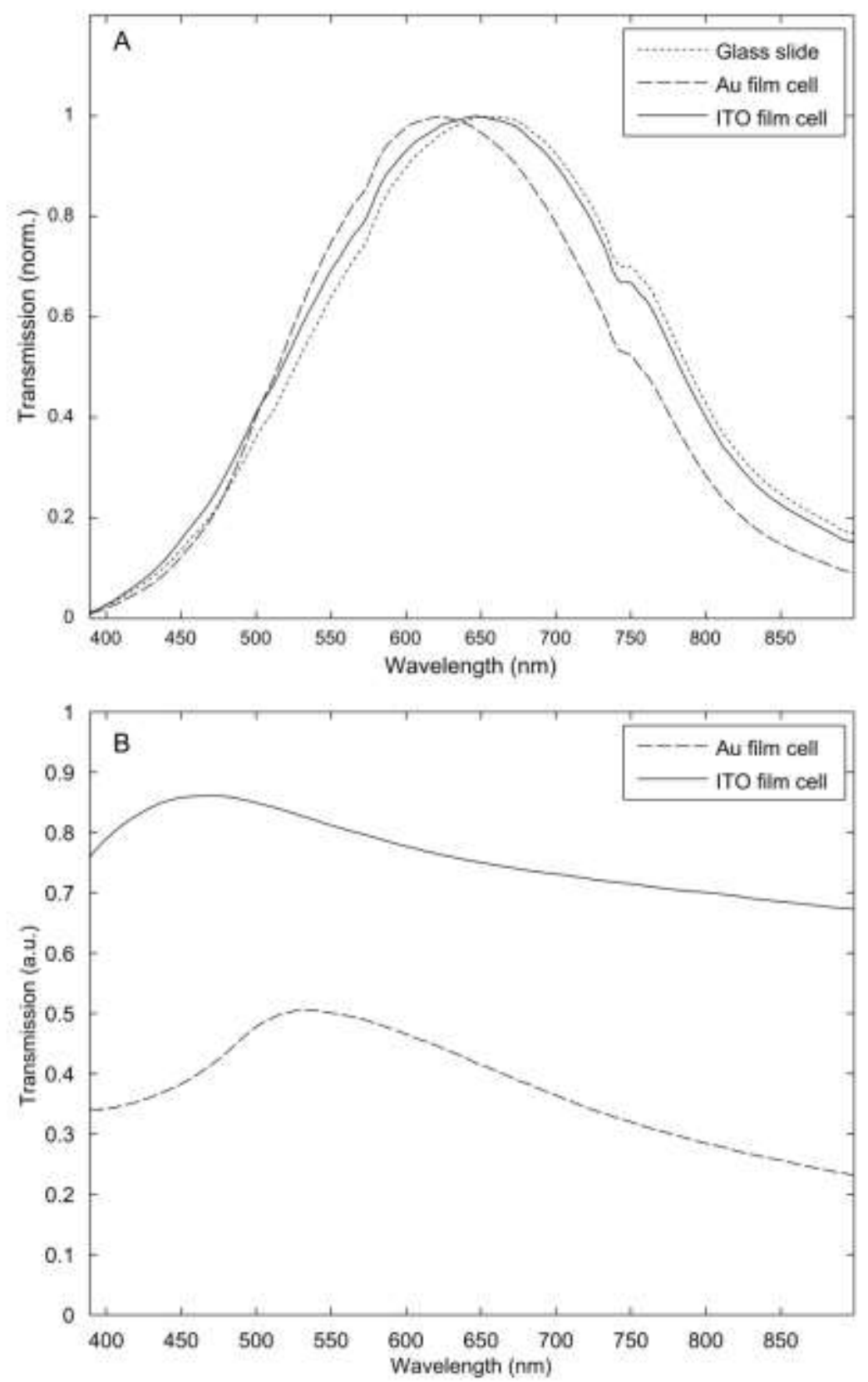

Figure S2. (A) Normalized transmission spectrum of a glass slide compared to electrochemical cells built using ITO or Au thin film working electrodes. All spectra were acquired using illumination from a $100 \mathrm{~W}$ tungsten-halogen lamp with normal incidence and were corrected by the CCD dark counts. (B) By dividing the dark-counts corrected normal incidence transmission spectra of each cell by the glass slide spectrum in A, the flat-field transmission spectra of the thin film and electrolyte was found. Identical collection conditions were used for the Au thin film and ITO thin film electrochemical cells, but the much higher intensity transmission of the glass slide required a lower CCD exposure time. Hence, the units for transmission are arbitrary. Direct numerical comparison between Au film cell and ITO film cell transmission is valid, but absolute transmission cannot be calculated using this experimental setup. 


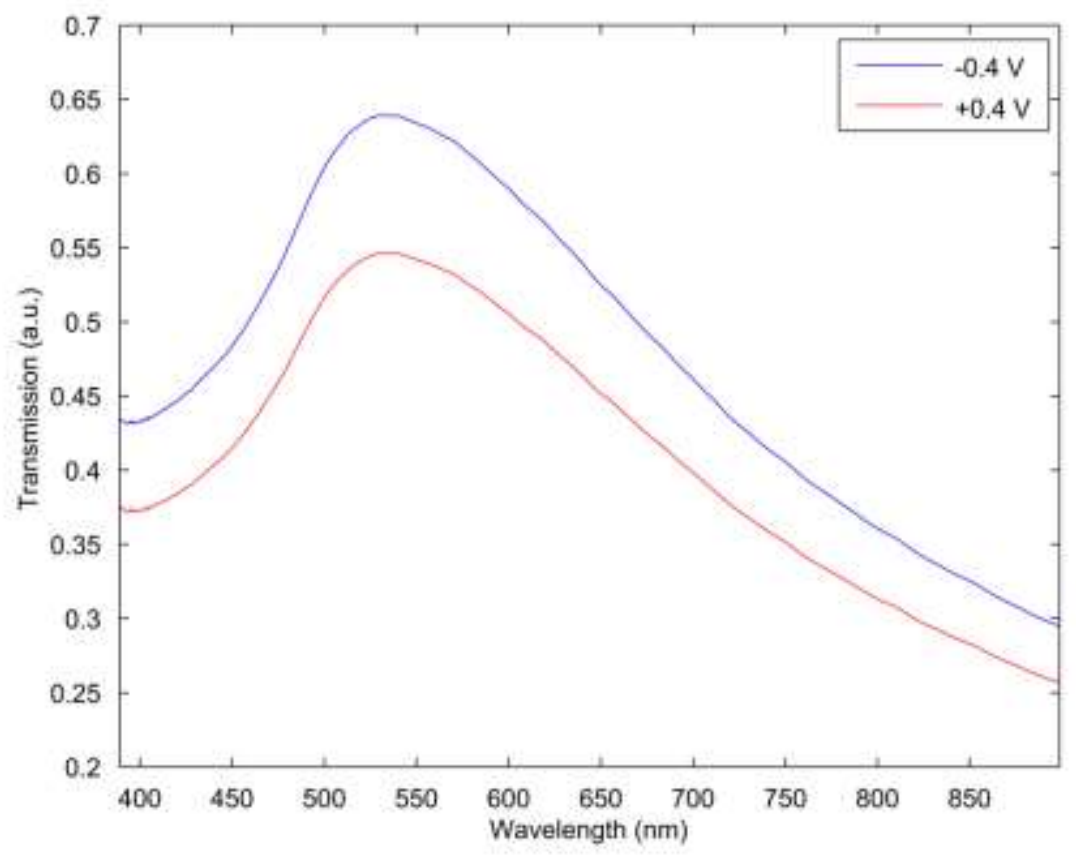

Figure S3. Transmission spectrum of an electrochemical cell built with $\mathrm{Au}$ film and $100 \mathrm{mM} \mathrm{NaSO}_{4}$ as a function of applied potential. The transmission spectrum through an Au film electrochemical cell was collected at both -0.4 and 0.4 V. Dark counts were subtracted from both spectra and were subsequently divided by the lamp standard as described in Figure S2. For reference, typical resonance wavelengths for each mode on the Au film are as follows: Au nanoparticle monomers/Au film low energy bonding mode: $750 \mathrm{~nm}$, dipolar mode: $540 \mathrm{~nm}$. For Au nanoparticle dimers/Au film, screened bonding mode: $620 \mathrm{~nm}$, transverse mode: $525 \mathrm{~nm}$. See Figure S2 caption for details on the use of arbitrary units. 

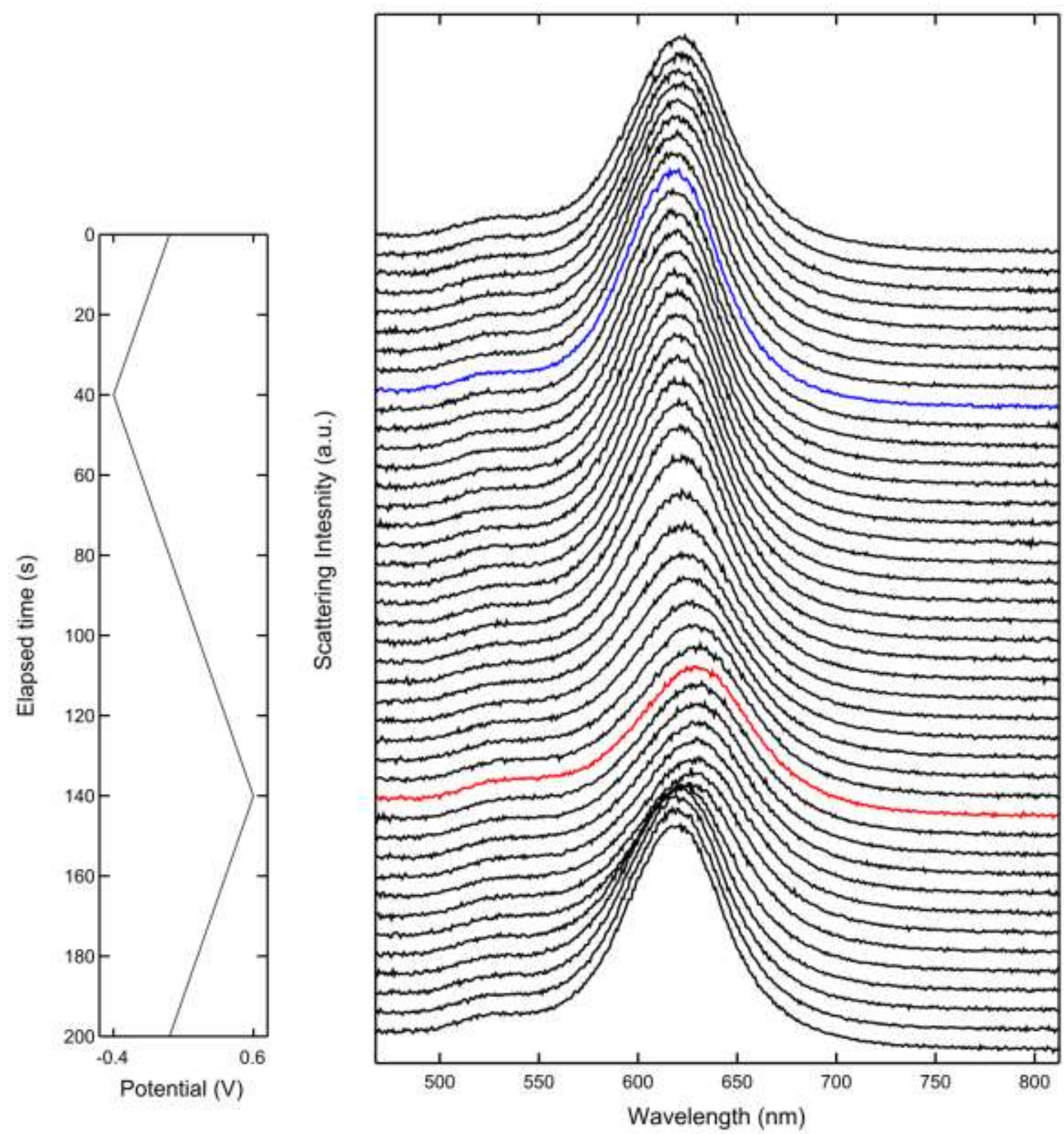

Figure S4. Scattering spectrum of a dimer on Au film during CV in $100 \mathrm{mM} \mathrm{Na}_{2} \mathrm{SO}_{4}$. As shown in Figure 2 of the main text, conductively bridged dimers showed a large response to sulfate adsorption. In this waterfall plot, the beginning of the cycle is shown as the top spectrum. For clarity, the spectrum acquired at $-0.4 \mathrm{~V}$ is colored blue and the spectrum acquired at $0.6 \mathrm{~V}$ is colored red. 

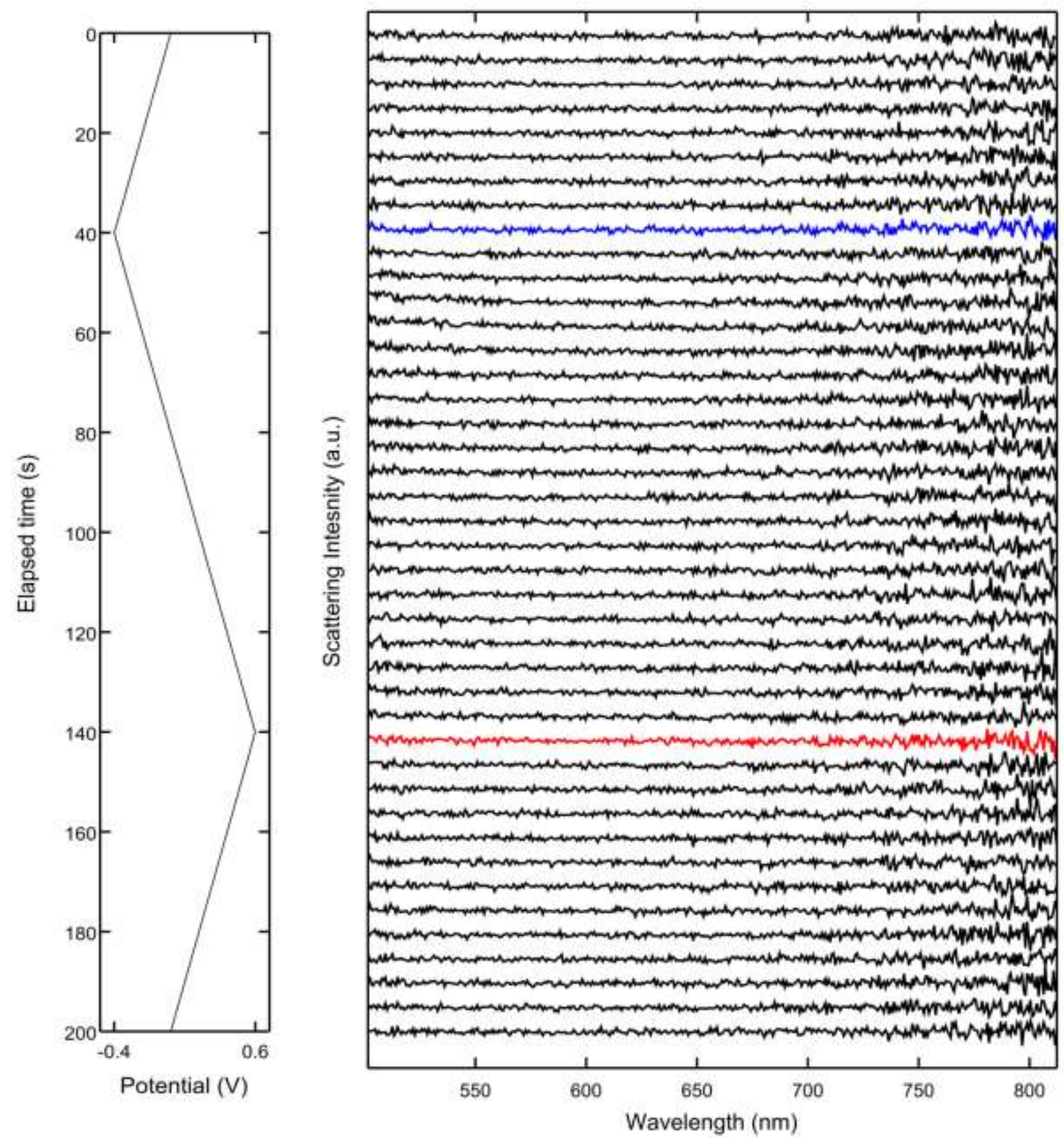

Figure S5. Scattering spectrum of Au film background during $\mathrm{CV}$ in $100 \mathrm{mM} \mathrm{Na}_{2} \mathrm{SO}_{4}$. A background scattering spectrum was collected at an area with Au film but no nanoparticles. Then a series of scattering spectra were collected at another nearby area, also with no nanoparticles. The series of scattering spectra (right) as a function of potential (left) show that the background scattering does not change a measurable amount. This allowed us to use a single background spectrum acquired at open circuit potential for background subtraction for all spectra in the entire single-nanoparticle CV experiment. A new background was taken before the measurement of each nanoparticle as a precaution against any film inhomogeneity. In this waterfall plot, the beginning of the cycle is shown as the top spectrum. For clarity, the spectrum acquired at $-0.4 \mathrm{~V}$ is colored blue and the spectrum acquired at $0.6 \mathrm{~V}$ is colored red. 


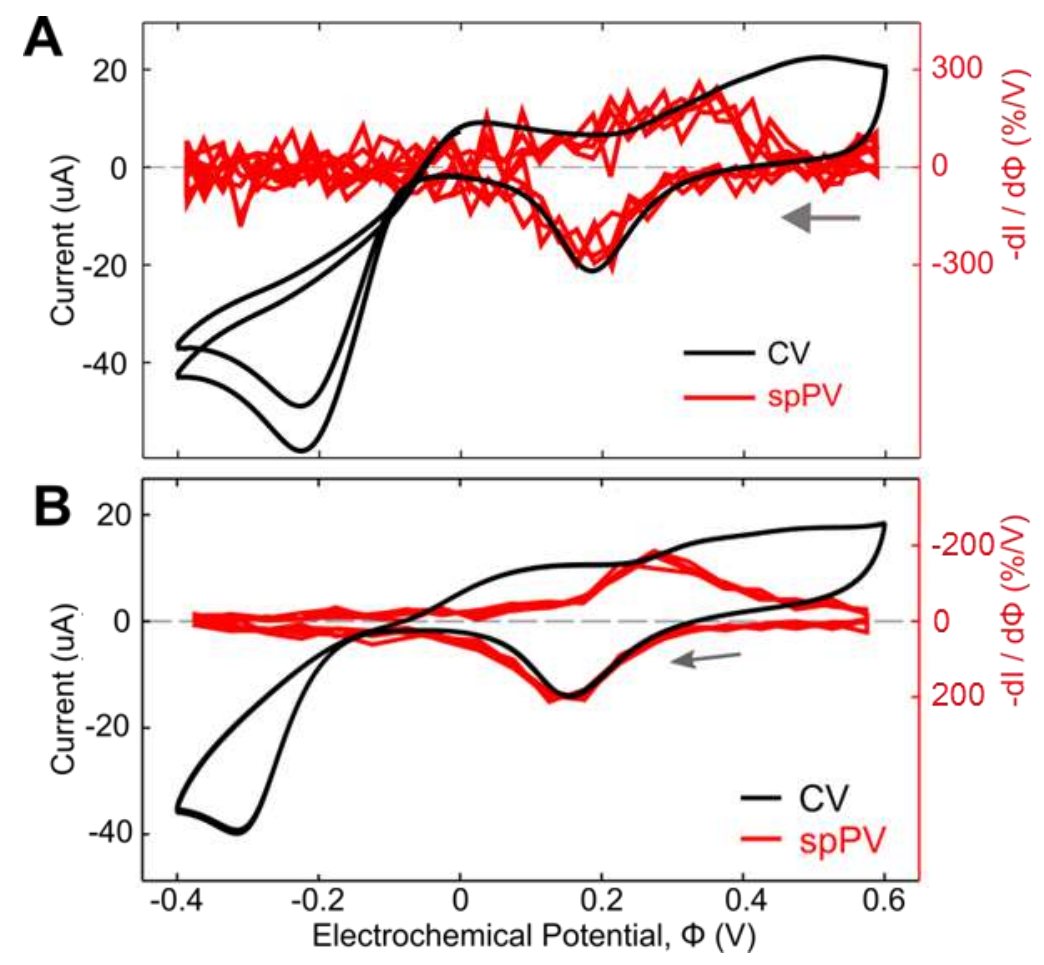

Figure S6. Effect of $\mathrm{pH}$ on acetate adsorption at (a) $\mathrm{pH} 12$ and (b) $\mathrm{pH} 8.5$ for spPV and bulk CV. Using the same experimental setup and data analysis as Figure 3A, the $\mathrm{pH}$ of acetate electrolyte was varied by the addition of $\mathrm{NaOH}$ to reach $\mathrm{pH}$ 12. The spPV and bulk $\mathrm{CV}$ signal was similar for both $\mathrm{pH} 8.5$ and $\mathrm{pH}$ 12 , confirming that spPV is primarily reporting on acetate adsorption under conditions in which acetate is fully deprotonated. 


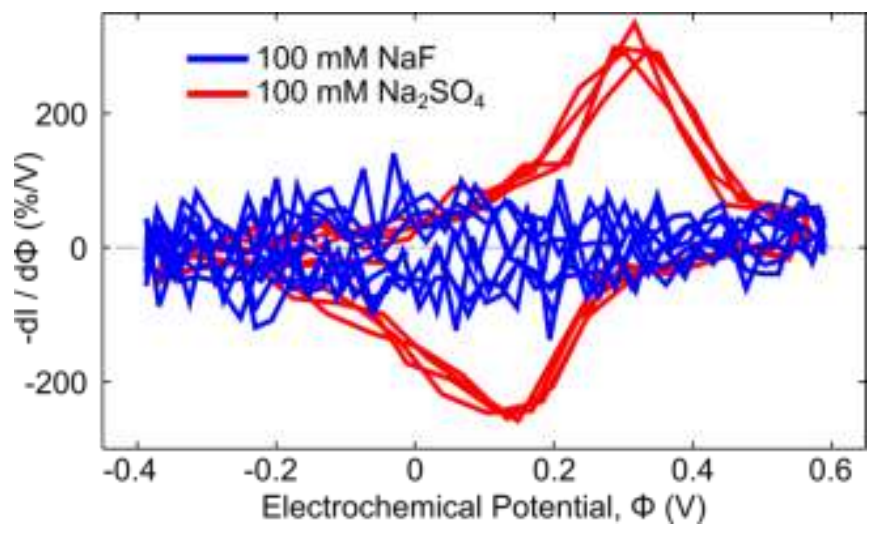

Figure S7. Sample spPV trace from $\mathrm{F}^{-}$control experiment compared to spPV response to sulfate adsorption. Identical samples were prepared (50 nm dimer on $20 \mathrm{~nm}$ Au film) and placed in a $100 \mathrm{mM}$ $\mathrm{Na}_{2} \mathrm{SO}_{4}$ vs inert $100 \mathrm{mM} \mathrm{NaF}$ spectroelectrochemical cell. Over the same potential range, no spPV signal was detected for $100 \mathrm{mM} \mathrm{NaF}$, indicating no signal from fluoride electroadsorption. Also, Au oxidation and hydrogen nucleation do not contribute to the observed spPV signal. 
— Integrated intensity (-d $\Sigma \mid / d \Phi)$

— SB mode intensity (-dl / d $\Phi)$
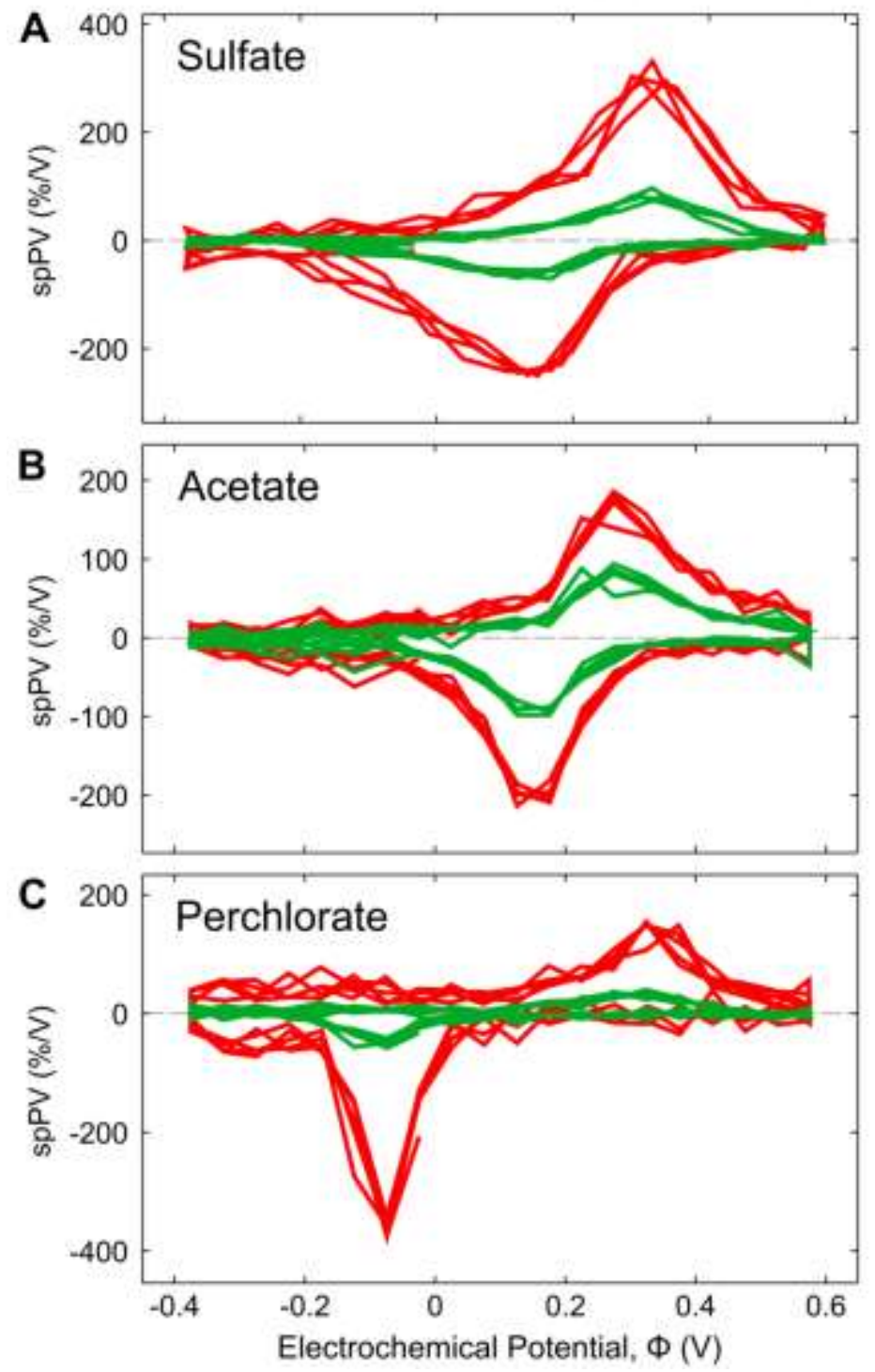

Figure S8. Comparison between spPV signals derived from SB mode intensity $v s$. integrated scattering intensity. spPV based on the SB mode intensity had much higher modulation depth than that of the integrated intensity. As visible in Figure 2A and 2B, the SB mode intensity responds strongly to anion adsorption while the T mode responds very weakly. Though the SB mode has a larger spectral weight, the T mode still contributes a significant amount of scattered light. Because the integrated scattering intensity includes this light from the T mode, the relative modulation depth is decreased. The differences between the two spPV variations can be seen for the cases of (A) sulfate adsorption (B) acetate adsorption, and (C) perchlorate adsorption. 

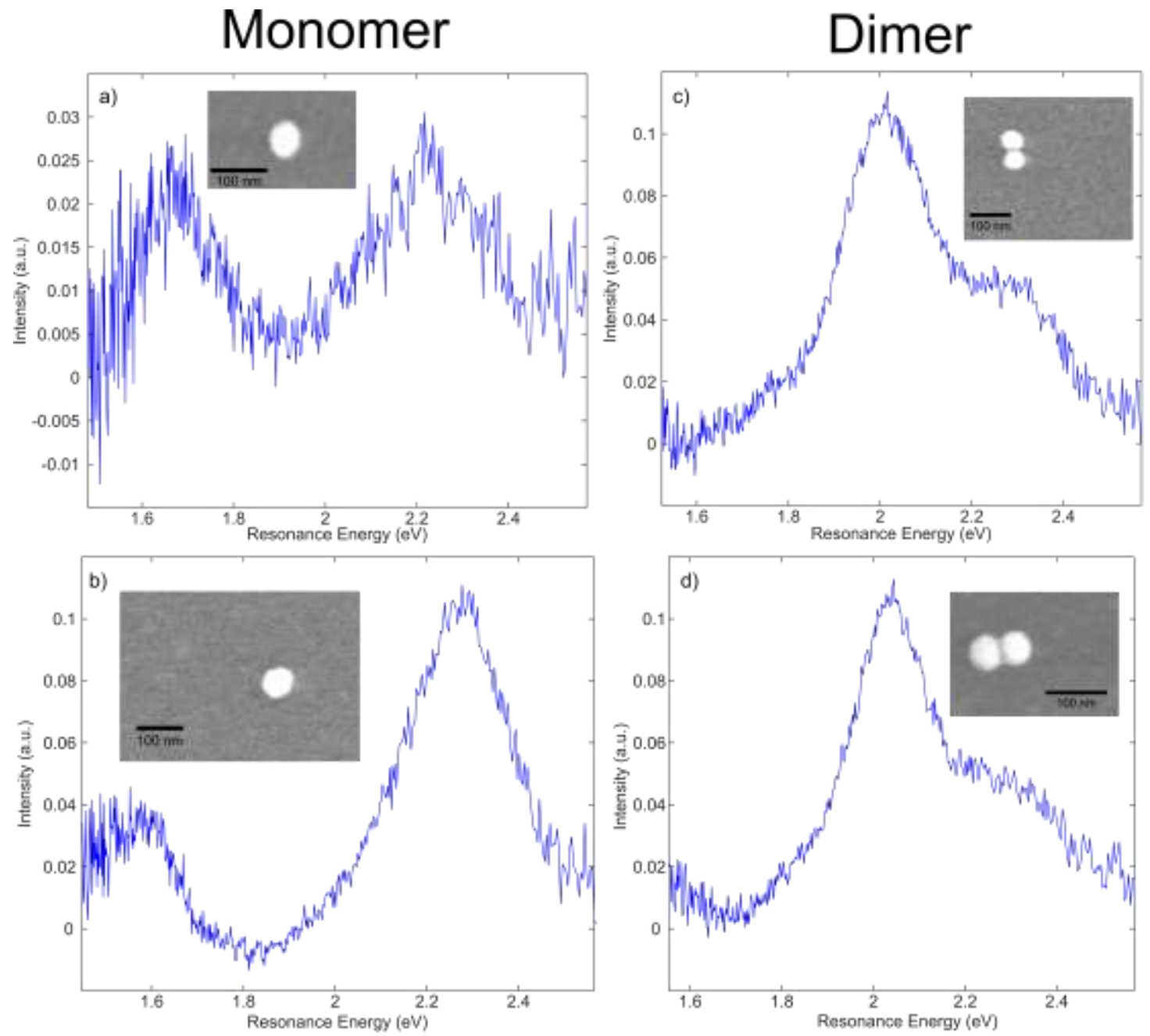

Figure S9. The spectra of single $50 \mathrm{~nm}$ Au nanoparticles (a, b) and their dimers (c, d) on Au film with correlated SEM (insets) to demonstrate that single particles and dimers can be distinguished spectrally. 